\title{
A Systematic Review of the Stability of Extemporaneous Pediatric Oral Formulations
}

\author{
Anteneh Belayneh (iD and Zenaw Tessema \\ Department of Pharmacy, College of Health Sciences, Debre Markos University, Debre Markos, Ethiopia \\ Correspondence should be addressed to Anteneh Belayneh; antejour32@gmail.com
}

Received 29 September 2021; Revised 26 November 2021; Accepted 4 December 2021; Published 15 December 2021

Academic Editor: Carlo Eduardo Medina-Solis

Copyright ( 2021 Anteneh Belayneh and Zenaw Tessema. This is an open access article distributed under the Creative Commons Attribution License, which permits unrestricted use, distribution, and reproduction in any medium, provided the original work is properly cited.

\begin{abstract}
Background. Extemporaneous compounding is a pharmacy practice to produce suitable pharmaceutical preparations when there are no commercially available, licensed, and age-specific dosage forms. Compared to the use of authorized drugs, these preparations have significant risks. Stability issues are one of the major concerns during the preparation of extemporaneous formulations. Aim. The aim of this work was to study the stability of pediatric extemporaneous formulations of commercially available conventional solid dosage forms by reviewing systematically the currently available stability studies. Method. Articles were searched in the databases of the Web of Science, PubMed, Scopus, EMBASE, Cochrane Library, and Google Scholar. From all the searched articles, a total of 28 experimental studies reporting the stability of oral pediatric extemporaneous formulations were included based on the inclusion criteria. Oral extemporaneous formulations from commercially available dosage forms and pure drugs were considered. According to the United States and British Pharmacopeia (USP and BP), most extemporaneous formulations are accepted as chemically stable if they maintain $\geq 90 \%$ of the original drug amount, physically stable if there is no apparent change in physical property, and microbiologically stable if there is no growth of microorganisms in prepared formulations. Finding. In this study, most extemporaneous pediatric oral formulations were chemically, physically, and microbiologically stable and retained more than $90 \%$ of the initial content. Very few studies did not include either a physical stability test or a microbiological stability test. Conclusion. According to this systematic review, the chemical and physical instabilities as well as microbial growth on pediatric oral extemporaneous formulations are very rare in published experimental studies. Most studies show that extemporaneous preparations are stable at the ICH recommended storage conditions and duration. Generally, extemporaneously prepared oral formulations will be the promising option for child medications.
\end{abstract}

\section{Introduction}

1.1. Background. The shortage of suitable pharmaceutical dosage forms specifically designed for children is the major challenge for pediatric treatment. Most FDA-approved adult pharmaceutical dosage forms are not appropriate formulations for pediatric use. The lack of sufficient information on pediatric administration often leads to the unauthorized use of adult preparations by healthcare professionals [1]. In some cases, there are no licensed or substitute products that fully meet the clinical needs of specific patients, so it is necessary to temporarily prepare a limited number of customized products for individual patients. About 15\% to $80 \%$ of all drugs used by hospitalized children are either unlicensed or used outside of the product's license specifications ("off-licence") [2].

Extemporaneous formulation describes the use of traditional compounding techniques by pharmacists to manipulate various drugs and chemical ingredients to produce suitable drugs when commercial forms are not available. These techniques are widely used in the practice of pediatric pharmacy. Most approved oral medications for adults are provided in tablet or capsule form, usually in a single adult dose form or in a liquid form that is not suitable for infants. However, the dose size of pediatric medications should change proportionally to body surface area and body weight during childhood. Also, most of the pediatric population cannot swallow pills, capsules, and other conventional dosage 
forms. To prevent the inappropriate use of unlicensed and unapproved adult medications, pharmacists will prepare suitable pediatric preparations [3]. This can be accomplished by grinding approved adult solid dosage forms such as tablets or by using capsule contents (powder and granules). Then, the powder can be prepared in the form of oral solution or suspension preparations using appropriate excipients and a suitable vehicle to produce, or it can be diluted into lower strength solid dosage forms using inert diluents. Sometimes the tablets are segmented into lower portions (half or a quarter) to get a suitable dosage unit for children [2].

\subsection{Stability of Extemporaneous Pediatric Formulation.} The physical, chemical, and microbiological stability should be considered during the quality assessment of extemporaneous preparation. It is very important to meet the storage conditions indicated on the label. Even if it has been proven that a given pharmaceutical preparation has sufficient physical, chemical, and microbiological stability, the bioavailability and palatability of the formulation may not be proven. Few pharmaceutical formulations are supported by evidenced data that determine sufficient absorption curves and/or bioequivalence with licensed formulations. Insufficient access to raw materials and equipment is also another concern during the compounding of good quality extemporaneous pharmaceutical products. In order to reduce degradation and deterioration, the maximum shelf life of the product is 28 days, unless the product is chemically unstable, so the shelf life is based on the stability of the respective products. Stability studies of these formulations are usually conducted in a short period of time. The lack of stability data limits the availability of many pediatric drugs. The candidate formulations available for extemporaneous preparations are highly dependent on the accessibility of stability data and the ingredients required for the compounding [2].

The objective of this study was to systematically review the stability of pediatric extemporaneous pharmaceutical formulations. The specific aim of this study was first to assess the stability of oral pediatric extemporaneous formulations by reviewing the currently available experimental literature and to provide evidence-based or best practice guidance about the chemical, physical, and microbiological stability of extemporaneous oral preparations of medicines for pediatrics. This helps policy makers and clinical practitioners who use extemporaneous preparations for pediatrics. Pharmacists, the main concerned professionals of pharmaceutical compounding, will benefit from the findings of this systematic review.

\section{Methods}

2.1. Literature Searching Strategy. The systematic reviews follow the Cochrane Collaboration guidelines, and we record the results according to the PRISMA guidelines for systematic reviews and meta-analysis preferred reporting project (PRISMA flowchart) [4]. We searched related experimental works of literature according to the study objectives from reliable databases of the Web of Science, PubMed, Scopus, EMBASE, Cochrane Library, and Google Scholar databases, written in English from June 1, 2021, to July 5, 2021. We combined the search strategy for free text terms and exploited the MESH title for the topics "Extemporaneous formulation OR Extemporaneous preparation OR Extemporaneous compounding," "stability," and "Peadiatric OR child OR Neonate OR Infant" using the Boolean operators like "AND” or "OR."

2.2. Study Selection and Eligibility Criteria. All currently online available experimental works conducted on the stability of extemporaneous pediatric formulations were included in the study. The articles and records which had no stability data, not focused on pediatric formulations, not focused on oral formulations, and articles without an informative abstract or full document were excluded from the study.

\subsubsection{Eligibility Criteria}

(1) Inclusion Criteria. Two researchers (AB and ZT) independently and carefully reviewed the content of each retrieved article. Finally, documents that meet the following criteria are included in the study.

Population: studies on the stability of pediatric extemporaneous formulations were included

Study area: all articles were included irrespective of the specific study area and year of the study

Study design: original experimental works of literature which have data on the stability of pediatric extemporaneous preparations were eligible

Language: documents published in English were considered

Publication condition: documents that fulfill the inclusion criteria were considered regardless of their publication status

(2) Exclusion Criteria. Two independent reviewers performed blind data extraction after evaluating the abstract and full text of the literature. After reading the full text and abstract, articles with methodological issues were excluded by two independent researchers. Due to incomplete data, inaccessible full-text articles were not included in the review.

(3) Data Extraction. Using the previously tested data extraction format, the researchers extracted the necessary data. Data extracted from included studies are as follows: author, study area, method of chemical stability test, source drug for extemporaneous formulation, storage condition of extemporaneous formulation, chemical stability result, physical stability result, and microbiological stability study. Any differences between the two authors on data extraction are resolved through discussion.

\section{Results and Discussion}

We reviewed all available studies which focused on the chemical, physical, and microbiological stability of pediatric oral extemporaneous formulations. We considered whether 


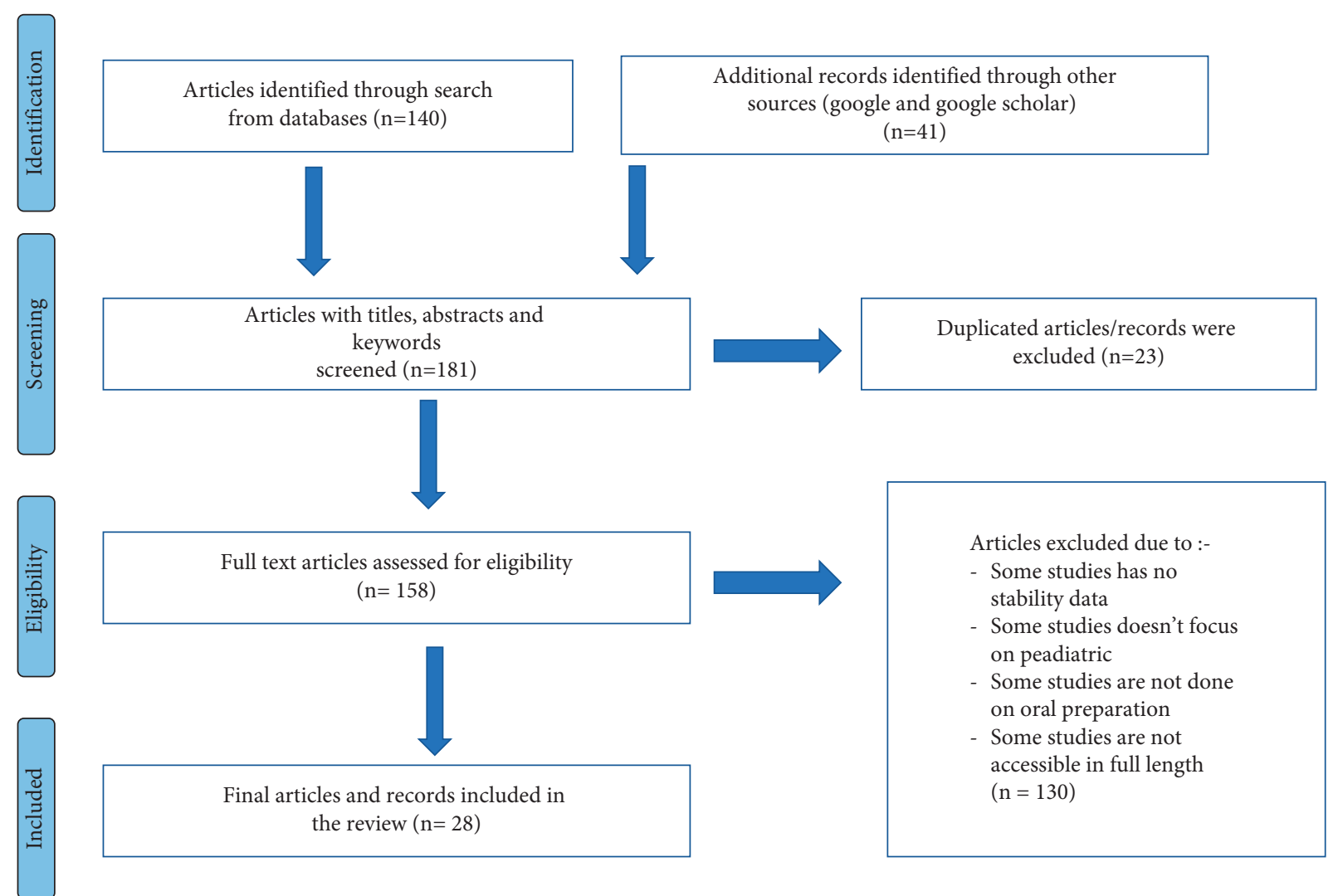

FIGURE 1: PRISMA diagram showing studies utilized for systematic review of stability of pediatric oral extemporaneous oral formulations.

or not instability problems occurred in such preparations. A limitation of our review is that the protocol was not previously registered. We found encouraging research on the stability of many drugs for pediatric oral formulations.

3.1. Selection of Included Studies. The search for the database has brought a total of 181 research items. Duplicate research $(n=23)$ was eliminated through its titles and summaries. The research approved by the abstract review was also examined with its title. Finally, a total of 28 studies (experimental articles) were included in this systematic review (Figure 1).

\subsection{Source of Pediatric Extemporaneous Oral Liquid} Formulations. In this systematic review, commercially available tablets and capsules are the most commonly used sources for pediatric extemporaneous preparations. Pure drugs, injectable preparations, and pellets are also used as sources of pediatric extemporaneous preparations. The results are consistent with other studies (Table 1). Research conducted at the Malta Hospital shows that most improvised pediatric compounding is made by converting capsules and tablets into oral liquids or powder. Others are made from active ingredients in bulk, such as oseltamivir powder to make an oseltamivir phosphate solution [2]. Preparation of children's oral medicines is subject to much variation in hospitals throughout Europe, and there is little harmonization of formulations or information on the stability of products. The European Union could be the focus for improving the availability of appropriate authorized medicines for children and ensuring that when extemporaneous preparation is necessary, it is of a common high standard [3].

3.3. Methods for Stability Testing. For this study, high-performance liquid chromatography (HPLC) is the frequently used method to check the chemical stability of most extemporaneous preparations. The UV-spectrophotometer and other methods are also used rarely to check the stability of these preparations (Table 1). HPLC and UV-spectrophotometer are the recommended methods for stability test of pharmaceutical formulations by all pharmacopeias including United States Pharmacopeia (USP) and British Pharmacopeia (BP) [7]. The stability of the peaks of the analyte and the degradation product that are completely separated from each other is indicative of the chromatogram of the HPLC method. [8].

\subsection{Storage Condition of Pediatric Extemporaneous}

Formulations. During storage, pharmaceuticals are prone to physical and chemical degradation. These degradations may change the pharmacological properties of the drug, reducing its benefits and increasing its harmful effects. The physical factors which affect the stability of the drug are light, solvent, heat, oxygen, and humidity [9].

In this review, most of the pediatric extemporaneous formulations were stored at temperatures of 4,25 , and $40^{\circ} \mathrm{C}$ with and without light before testing. Some were stored at 


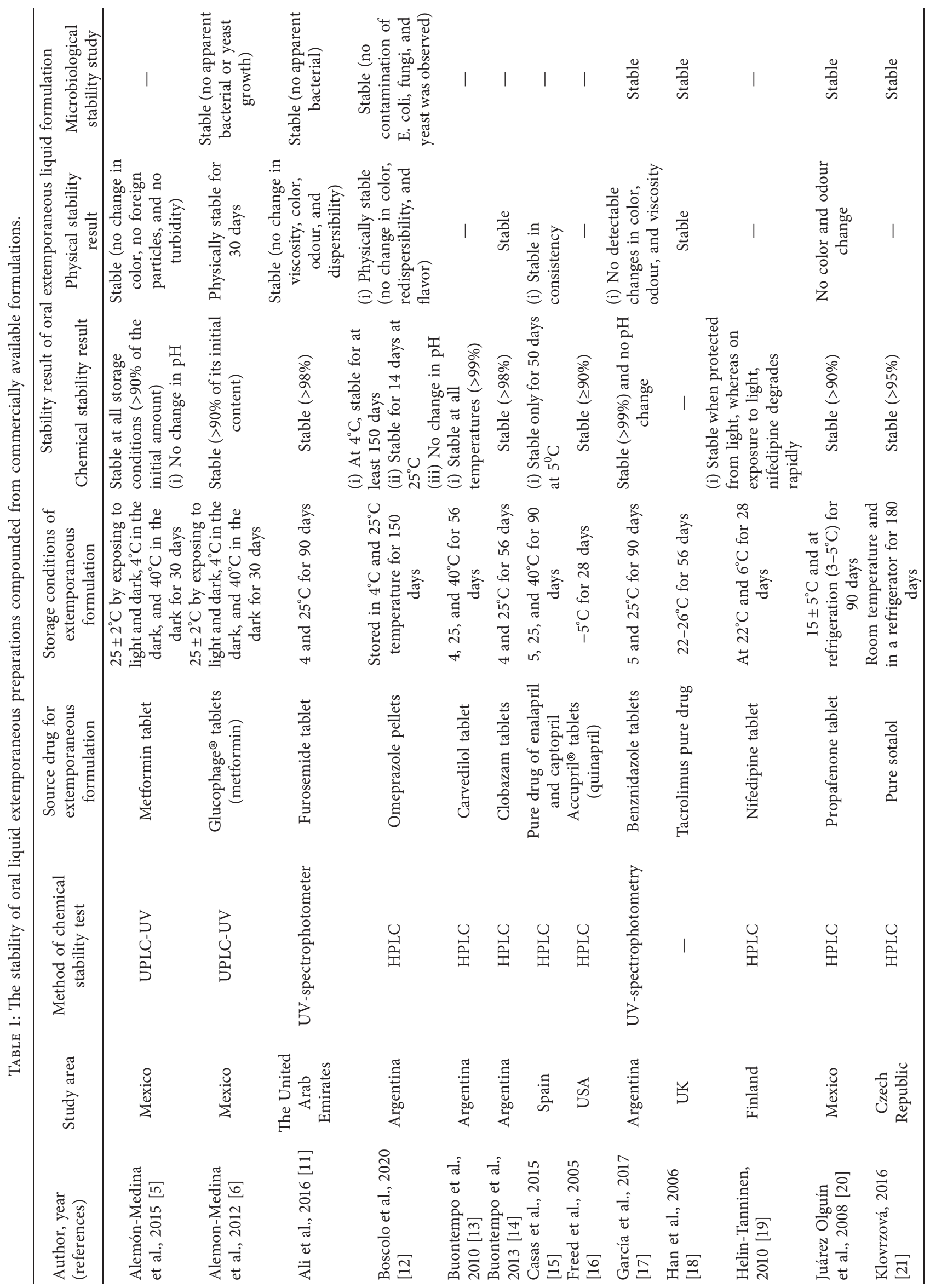




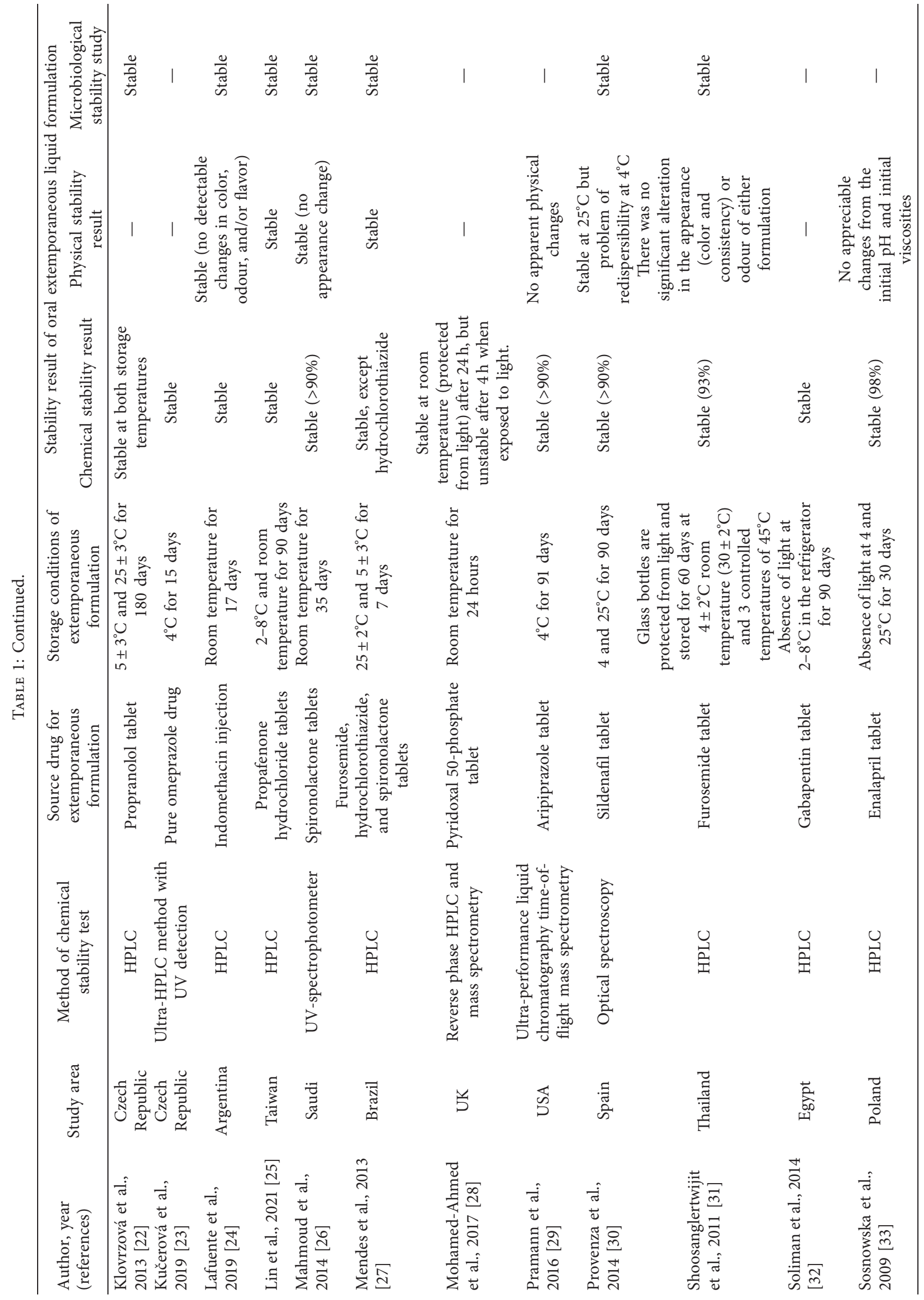




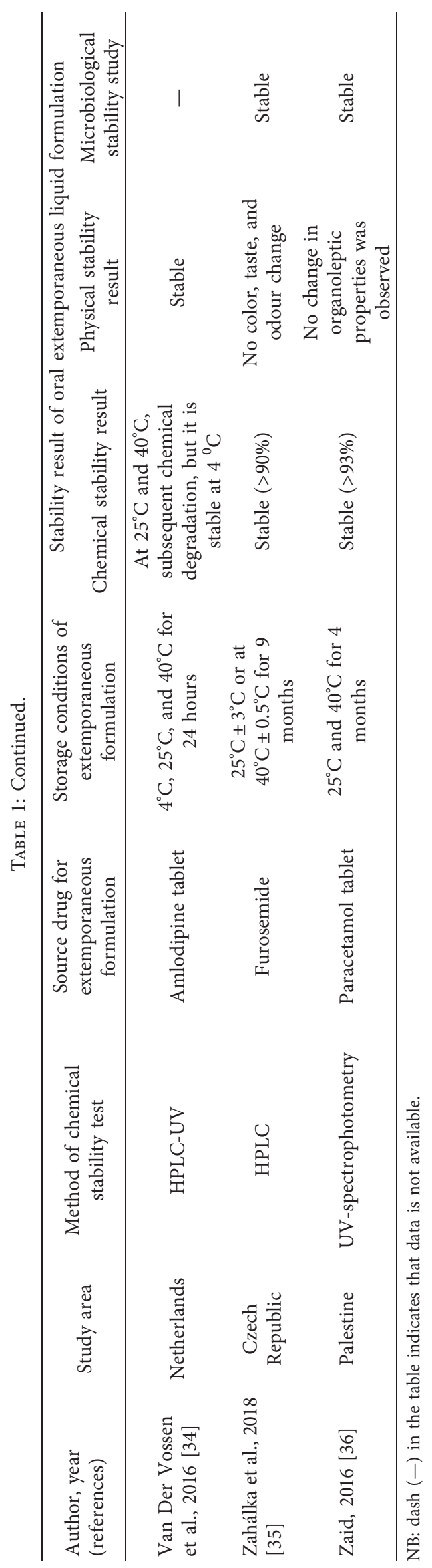


room temperature until they were tested. The storage duration for the test was varied from 24 hours to 150 days (Table 1). All storage conditions comply with the ICH guideline which focuses on the storage conditions of pharmaceuticals for the purpose of stability testing of APIs [10].

\subsection{Chemical Stability of Pediatric Oral Extemporaneous} Liquid Formulations. Stability studies to ensure pharmaceutical product safety, quality, and efficacy are preserved throughout the shelf life and are considered as a precondition for approval of any pharmaceutical preparations. Experimental stability studies should be done in a wellorganized manner according to the World Health Organization (WHO) and International Conference on Harmonization (ICH) guidelines. Stability refers to the degree to which a product maintains the same characteristics during its storage and use within the specified limits. Each drug maintains chemical integrity and labeled efficacy within the specified range [8].

More than $96 \%$ of pediatric oral extemporaneous liquid formulations in this review are stable at all storage conditions $\left(4^{\circ} \mathrm{C}, 25^{\circ} \mathrm{C}, 40^{\circ} \mathrm{C}\right.$, and room temperature). They retain more than $90 \%$ of their initial content of the active drug after the storage duration. But, some drugs are unstable in some specific conditions. For instance, nifedipine and pyridoxal phosphate oral liquid extemporaneous preparations were degraded in the exposure of light. Amlodipine was also degraded at 25 and $40^{\circ} \mathrm{C}$. From twenty-eight pediatric oral extemporaneous formulations, only three formulations showed chemical degradation (Table 1).

The USP, BP, and European Pharmacopeia have established that the acceptable range of most compounded preparations is typically $\pm 10 \%$, or within the range of $90.0 \%$ $110.0 \%$. Even for some drugs, if they retain $85 \%$ of their original content, it is acceptable [8].

\subsection{Physical Stability of Pediatric Oral Extemporaneous} Liquid Formulations. The appearance, consistency, uniformity of content, solution clarity, moisture content, particle size and shape, $\mathrm{pH}$ value, and integrity of pharmaceutical packaging may change, which may affect its stability. Such physical changes can be caused by shock, vibration, wear, and temperature fluctuations (such as freezing, thawing, or shearing) [37]. To conclude a pharmaceutical product as physically stable, the original physical properties, including appearance, palatability, uniformity, dissolution, and suspendability, must be retained [8].

In this review, almost all (98.9\%) of the extemporaneous pediatric formulations are physically stable at all storage conditions. There were no apparent changes in color, odour, viscosity, redispersibility, and $\mathrm{pH}$. But some of the literature (7 from 28) have no data for physical stability (Table 1).

3.7. Microbiological Stability of Pediatric Oral Extemporaneous Liquid Formulations. The stability of a pharmaceutical product can also be affected because of microbiological changes such as the growth of microorganisms in nonsterile products and changes in preservative efficacy. Microbiological tests include sterility, preservative efficacy, and microbial count as applicable. Resistance to microbial growth is retained according to the specified requirements [37].

We also discuss the factors that affect microbial contamination in popular dosage forms (e.g., tablets, sterile products, cosmetics). When these products are contaminated, the microorganisms can cause changes. The effects range from mild changes (e.g., discoloration, texture alteration) to severe effects (e.g., changes in activities, toxicity). In this study, most pediatric oral extemporaneous preparations in this review are stable (no growth of microorganisms at the storage temperature during the storage conditions). But some studies do not include microbiological stability study (Table 1)).

\section{Conclusion}

There are severe shortage of commercial drugs suitable for children, due to this there is a need for extemporaneous oral preparations. Stability study data are very crucial for hospital pharmacists to confirm the safety and quality of the dispensed extemporaneous preparations, especially for pediatric patients. To be used as an alternative for commercial products and to be therapeutically safe and effective, the extemporaneous pharmaceutical preparation must be physically, chemically, and microbiologically stable.

According to this systematic review, several experimental studies showed that the chemical and physical instabilities and microbial growth on pediatric oral extemporaneous formulations are very rare. Most studies revealed extemporaneous preparations are stable at the $\mathrm{ICH}$ recommended storage conditions and duration. It is recommended that an expiry date of a maximum of one month (or less if advised in the published study or if antimicrobial preservatives cannot be used) is applied to all extemporaneous formulations. This will encourage to use regular fresh preparation and help to assure effectiveness and safety. It also allows the practitioner to regularly review the patient's use of the preparation.

Generally, extemporaneously prepared oral formulations (medicines) from commercially available tablets, capsules, powders, and other dosage forms will be the promising option for pediatrics.

\section{Data Availability}

The data used to support the findings of this study are included within the article.

\section{Conflicts of Interest}

The authors declare that they have no conflicts of interest.

\section{Authors' Contributions}

All authors made a significant contribution to the work reported, whether that was in the conception, study design, execution, acquisition of data, analysis, and interpretation, 
or in all these areas; took part in drafting, revising, or critically reviewing the article; gave final approval of the version to be published; agreed on the journal to which the article has been submitted; and agreed to be accountable for all aspects of the work.

\section{References}

[1] A. Belayneh, E. Tadese, and F. Molla, "Safety and biopharmaceutical challenges of excipients in off-label pediatric formulations," International Journal of General Medicine, vol. 13, pp. 1051-1066, 2020.

[2] A. Aquilina, "The extemporaneous compounding of paediatric medicines at Mater Dei Hospital," Journal of the Malta College of Pharmacy Practice, vol. 7, no. 19, 2013.

[3] F. Brion, A. Nunn, and A. Rieutord, "Extemporaneous (magistral) preparation of oral medicines for children in European hospitals," Acta Paediatrica, vol. 92, no. 4, pp. 486-490, 2003.

[4] A. Liberati, D. G. Altman, J. Tetzlaff et al., "The PRISMA statement for reporting systematic reviews and meta-analyses of studies that evaluate health care interventions," Explanation and Elaboration, vol. 62, no. 10, pp. e1-e34, 2009.

[5] R. Alemón-Medina, J. L. Chávez-Pacheco, L. Rivera-Espinosa et al., "Extemporaneous formulations of metformin for pediatric endocrinology: physicochemical integrity, cytotoxicity of sweeteners, and quantitation of plasma levels," Clinical Therapeutics, vol. 37, no. 8, pp. 1689-1702, 2015.

[6] R. Alemon-Medina, R. Coria-Jimenez, B. Ramirez-Mendiola et al., "Physicochemical and microbiological stabilities of a sweetened and calorie-free metformin extemporaneous formulation for pediatrics," Latin American Journal of Pharmacy, vol. 31, pp. 1253-1260, 2012.

[7] A. C. Cartwright, The British Pharmacopoeia, 1864 to 2014 : Medicines, International Standards and the State, Routledge, London, UK, 2016.

[8] L. V. Allen, G. S. Bassani, E. J. Elder, and A. F. Parr, "Strength and Stability Testing for Compounded Preparations," pp. 1-7, U.S. Pharmacopeia, Rockville, MD, USA, 2014.

[9] T. H. Mallhi, R. Khokhar, A. Khokhar, N. H. Alotaibi, and Y. H. Khan, "Stability studies of extemporaneous pharmaceutical products," in Drug Stability and Chemical Kinetics, pp. 237-246, Springer, Berlin, Germany, 2020.

[10] P. WECoSfP, Stability Testing of Active Pharmaceutical Ingredients and Finished Pharmaceutical Products, World Health Organization, Geneva, Switzerland, 2018.

[11] H. Ali, R. Saad, A. Ahmed, B. El-Haj, and P. Research, "Extemporaneous furosemide suspensions for pediatrics use prepared from commercially available tablets," Human Journals, vol. 5, no. 2, Article ID 116, 2016.

[12] O. Boscolo, F. Perra, L. Salvo, F. Buontempo, and S. Lucangioli, "Formulation and stability study of omeprazole oral liquid suspension for pediatric patients," Hospital Pharmacy, vol. 55, no. 5, pp. 314-322, 2020.

[13] F. Buontempo, E. Bernabeu, R. J. Glisoni, E. Quiroga, C. Bregni, and D. Chiappetta, "Carvedilol stability in paediatric oral liquid formulations," Farmacia Hospitalaria, vol. 34, no. 6, pp. 293-297, 2010.

[14] F. Buontempo, M. A. Moretton, E. Quiroga, and D. A. Chiappetta, "Extemporaneous clobazam suspensions for paediatric use prepared from commercially available tablets and pure drug," Farmacia Hospitalaria, vol. 37, no. 2, 2013.

[15] M. Casas, J. Ảlvarez, and M. Lucero, "Physicochemical stability of captopril and enalapril extemporaneous formulations for pediatric patients," Pharmaceutical Development and Technology, vol. 20, no. 3, pp. 271-278, 2015.

[16] A. L. Freed, S. B. Silbering, K. J. Kolodsick, D. T. Rossi, M. Mahjour, and C. A. Kingsmill, "The development and stability assessment of extemporaneous pediatric formulations of Accupril," International Journal of Pharmacy, vol. 304, no. 1-2, pp. 135-144, 2005.

[17] M. C. García, R. H. Manzo, and A. F. Jimenez-Kairuz, "Extemporaneous benznidazole oral suspension prepared from commercially available tablets for treatment of Chagas disease in paediatric patients," Tropical Medicine and International Health, vol. 20, no. 7, pp. 864-870, 2015.

[18] J. Han, A. Beeton, P. Long, I. Wong, and C. Tuleu, "Physical and microbiological stability of an extemporaneous tacrolimus suspension for paediatric use," Journal of Clinical Pharmacy and Therapeutics, vol. 31, no. 2, pp. 167-172, 2006.

[19] M. Helin-Tanninen, "Extemporaneous preparation of paediatric oral formulations: studies conducted in nifedipine powders, capsules and sunpensions in a hospital pharmacy," Licenciate Thesis, University of Eastern Finland, Kuopio, Finland, 2010.

[20] H. Juárez Olguín, C. Flores Pérez, B. Ramírez Mendiola, R. Coria Jiménez, E. Sandoval Ramírez, and J. Flores Pérez, "Extemporaneous suspension of propafenone: attending lack of pediatric formulations in Mexico," Pediatric Cardiology, vol. 29, no. 6, pp. 1077-1081, 2008.

[21] S. Klovrzová, L. Zahálka, T. Kř́iž et al., "Extemporaneous sotalol hydrochloride oral solutions for use in paediatric cardiology: formulation and stability study," European Journal of Hospital Pharmacy, vol. 23, no. 1, pp. 33-37, 2016.

[22] S. Klovrzová, L. Zahálka, L. Matysová, P. Horák, and Z. Sklubalová, "Pediatric oral solutions with propranolol hydrochloride for extemporaneous compounding: the formulation and stability study," Ceská a Slovenská Farmacie, vol. 62, no. 1, pp. 35-39, 2013.

[23] K. Kučerová, V. Reiská, F. Švec, L. K. Krčmová, and L. J. A. M. Matysová, "Fast determination of omeprazole in extemporaneous suspensions used in paediatrics and stability studies," Analytical Mathods, vol. 11, no. 4, pp. 517-523, 2019.

[24] Y. Lafuente, M. C. García, and A. Jiminez-Kairuz, "Extemporaneous indomethacin oral suspension prepared from injectable Ampules for therapy in premature infants and pediatric patients," International Journal of Pharmaceutical Compounding, vol. 23, no. 4, pp. 324-331, 2019.

[25] Y. S. Lin, S. C. Lin, Y. T. Hsieh et al., "Formulation and stability of an extemporaneously compounded propafenone hydrochloride oral suspension for children with arrhythmia," European Journal of Hospital Pharmacy, vol. 4, 2021.

[26] I. M. Mahmoud, Y. M. Ibrahim, M. A. Shakir, and A. El-Gied, "Extemporaneous preparations of pediatric oral formulations: stability studies conducted in spironolactone suspensions, powders and capsules in Saudi hospital pharmacies," Pharmaceutical Sciences, vol. 5, no. 2, pp. 1595-1602, 2014.

[27] C. Mendes, A. P. Costa, P. R. Oliveira, M. P. Tagliari, and M. Silva, "Physicochemical and microbiological stability studies of extemporaneous antihypertensive pediatric suspensions for hospital use," Pharmaceutical Development and Technology, vol. 18, no. 4, pp. 813-820, 2013.

[28] A. H. Mohamed-Ahmed, M. P. Wilson, M. Albuera, T. Chen, P. B. Mills, and E. Footitt, "Quality and stability of extemporaneous pyridoxal phosphate preparations used in the treatment of paediatric epilepsy," Journal of Pharmacy and Pharmacology, vol. 69, no. 4, pp. 480-488, 2017. 
[29] L. A. Pramann, L. W. Davidow, L. van Haandel, and R. Funk, "Development of extemporaneously compounded aripiprazole oral suspensions for use in children," International Journal of Pharmaceutical Compounding, vol. 20, no. 3, pp. 257-261, 2016.

[30] N. Provenza, A. Calpena, M. Mallandrich, L. Halbaut, and B. Clares, "Design and physicochemical stability studies of paediatric oral formulations of sildenafil," International Journal of Pharmaceutics, vol. 460, no. 1-2, pp. 234-239, 2014.

[31] J. Shoosanglertwijit, S. Kaewnopparat, B. Yongmaitreesakul, S. Pattayananthavej, and N. Kaewnopparat, "Physical, chemical, and microbiological stability of extemporaneous furosemide suspensions," Asian Biomedicine, vol. 5, no. 5, pp. 681-686, 2011.

[32] I. I. Soliman, E. A. Afify, and G. Al Shobragi, "Evaluation of extemporaneous pediatric gabapentin suspension prepared from gabapentin capsules," Latin American Journal of Pharmacy, vol. 33, no. 5, pp. 746-752, 2014.

[33] K. Sosnowska, K. Winnicka, and A. Czajkowska-Kosnik, "Stability of extemporaneous enalapril maleate suspensions for pediatric use prepared from commercially available tablets," Acta Poloniae Pharmaceutica, vol. 66, no. 321, Article ID e6, 2009.

[34] A. Van Der Vossen, I. Van Der Velde, O. Smeets et al., "Design and stability study of an oral solution of amlodipine besylate for pediatric patients," European Journal of Pharmaceutical Sciences, vol. 92, pp. 220-223, 2016.

[35] L. Zahálka, S. Klovrzová, L. Matysová, Z. Šklubalová, and P. Solich, "Furosemide ethanol-free oral solutions for paediatric use: formulation," HPLC method and stability study, vol. 25, no. 3, pp. 144-149, 2018.

[36] A. Zaid, "Extemporaneous compounding and stability evaluation of paracetamol-honey based syrup for pediatric use," Asian Journal of Pharmaceutics, vol. 10, no. 3, 2016.

[37] S. Bajaj, D. Singla, and N. Sakhuja, "Stability testing of pharmaceutical products," Journal of Applied Pharmaceutical Science, vol. 2, no. 3, pp. 129-138, 2012. 